Check for updates

Cite this: RSC Adv., 2017, 7, 33953

\title{
A computational study on hydrogen storage in potential wells using $\mathrm{K}$-intercalated graphite oxide
}

\begin{abstract}
Jaehyun Bae, ${ }^{\mathrm{ab}}$ Dongwook Kim, ${ }^{\mathrm{b}}$ Jong Hyun Jung (D) $^{\mathrm{b}}$ and Jisoon $\mathrm{Ihm}^{\text {*cb }}$
Using ab initio electronic structure calculations and grand canonical Monte Carlo simulations, we investigate the storage capacity of hydrogen molecules in a potential well created inside potassiumintercalated graphite oxide layers. We show that the binding energy of hydrogen located between layers of graphite oxide mainly originates from the dispersion interaction, and it is further increased slightly by induced dipole interactions. Its strength is fairly insensitive to the precise positions of the hydrogen molecules on the graphene plane, so the system may be described as a quasi-two-dimensional potential well. In this situation, the storage capacity is enhanced by the corresponding Boltzmann factor based on equilibrium thermodynamics. The trend of storage capacity with different geometries and chemical compositions of the scaffold materials is explained. With the present model, the density functional theory calculations and grand canonical Monte Carlo simulations predict a $2.5 \mathrm{wt} \%$ hydrogen storage capacity at room temperature at $10 \mathrm{MPa}$. For a model with increased potential depth, the storage capacity is predicted to increase up to $5.5 \mathrm{wt} \%$.
\end{abstract}

Received 8th May 2017

Accepted 17th June 2017

DOI: 10.1039/c7ra05173e

rsc.li/rsc-advances

density set by the US Department of Energy (DOE) by the year

\section{Introduction}

Hydrogen is considered to be a clean and sustainable future energy source, ${ }^{1}$ but storing hydrogen safely and efficiently under ambient conditions is a major obstacle in commercializing hydrogen fuel cell powered vehicles. Metal or chemical hydrides show high storage capacity ${ }^{2}$ but do not satisfy the requirements for the fast kinetics and reversibility. Metalorganic frameworks (MOFs) have been regarded as promising storage materials owing to their porosity, fast kinetics and reversibility, ${ }^{3,4}$ but high pressure and cryogenics are necessary for the adsorption process because of the weak adsorption energy of hydrogen molecules, mainly via van der Waals interactions $\left(\approx 0.05 \mathrm{eV} / \mathrm{H}_{2}\right)$. It has been found that the optimal binding energy for ambient condition storage $\left(\approx 0.3 \mathrm{eV} / \mathrm{H}_{2}\right)$ is realizable by introducing transition metal (TM) atoms into the MOFs. ${ }^{5,6} \mathrm{TM}$ atoms are bound to the linker part of the MOFs and $\mathrm{H}_{2}$ molecules are adsorbed to the TM atoms through the socalled Kubas interaction. ${ }^{7}$ However, because of their high cohesive energy and reactivity, TM atoms tend to form clusters and are easily contaminated by other gas molecules. This hinders the experimental realization of the target for storage

${ }^{a}$ Center for Correlated Electron Systems, Institute for Basic Science (IBS), Seoul 08826, Republic of Korea

${ }^{b}$ Department of Physics and Astronomy, Seoul National University (SNU), Seoul 08826, Republic of Korea

'Department of Physics, Pohang University of Science and Technology (POSTECH), Pohang 37673, Republic of Korea. E-mail: jihm1@postech.ac.kr; Fax: +82 54279 3099; Tel: +82542792065
2020 (5.5 wt\% and $\left.40 \mathrm{~g} \mathrm{~L}^{-1}\right)$.

Recently, it has been shown that a class of material mostly based on pillared graphene layers has large surface areas, and the magnitude of the hydrogen binding energy to the material is increased by functionalization and alkali metal ion doping. ${ }^{8-14}$ For example, Deng et al. proposed alkali-doped pillared carbon materials to maximize reversible $\mathrm{H}_{2}$ storage and suggested a possible scheme to synthesize practical storage materials. ${ }^{8}$ Dimitrakakis et al. used lithium-doped 3-dimensional carbon nanostructures as a storage material and showed that the induced dipole interaction between $\mathrm{H}_{2}$ and doped lithium increases the volumetric storage capacity significantly. ${ }^{9}$ Tylianakis et al. studied the hydrogen storage capacity of lithiumdoped graphite oxide, and explained that the storage capacity is closely related to the pore volume and the carbon to oxygen ratio of graphite oxide. ${ }^{10}$

In this study, we propose a storage mechanism based on the diffusive equilibrium between systems with different potentials and also suggest a storage material for the realization of this mechanism. The equilibrium condition of hydrogen inside and outside the material is $\mu_{\text {in }}=\mu_{\text {out }}$, where $\mu$ is the chemical potential of the hydrogen molecule. If the material can provide, to a first order approximation, a uniform potential well with a depth of $V(<0)$ relative to the outside, we will show later that the gas density in the potential well becomes $\mathrm{e}^{-\frac{V}{k_{\mathrm{B}} T}}$ times larger than that outside the potential well, ${ }^{15}$ where $T$ is the temperature and $k_{\mathrm{B}}$ is the Boltzmann constant. The exponential dependence of the density inside the material on $V$ allows for practical room temperature storage with a relatively small potential depth (e.g. 
the enhancement factor, $\mathrm{e}^{-\frac{V}{k_{\mathrm{B}} T}}$, turns out to be 100 for $V=$ $-0.12 \mathrm{eV}$ at $25^{\circ} \mathrm{C}$ ). Since an experimental technique for tailoring a strict 3-dimensional (3D) attractive potential for a non-polar molecule is not available in practice, we searched for 2-dimensional (2D) materials that interact with hydrogen molecules with the desired binding strength. For example, if the interlayer distance, $d$ (in the $z$-direction), of graphite is increased to twice the equilibrium distance between a $\mathrm{H}_{2}$ molecule and a singlelayer graphene, then $\mathrm{H}_{2}$ can penetrate between the layers and it interacts with both the upper and lower layers. In this situation, the van der Waals (dispersion) interaction energy between $\mathrm{H}_{2}$ and two graphene layers can reach $\approx-0.10 \mathrm{eV}$ and the extra binding energy of only a few tens of $\mathrm{meV}$ is enough for practical room temperature storage. Furthermore, the van der Waals interaction is almost independent of the $x y$ position of $\mathrm{H}_{2}$ on the graphene layer, so that the $\mathrm{H}_{2}$ molecules behave almost like a gas in 2D space as desired. ${ }^{16,17}$

To utilize the layered structure with enhanced $d$ derived from graphite, we suggest potassium intercalated graphite oxide (KGO) as a possible storage material. In a recent experiment, it was reported that the $d$ of graphite can be increased to $6-7 \AA$ by oxidation, and further increased to $1 \mathrm{~nm}$ or more by potassium intercalation. ${ }^{18}$ In the experiment, graphite oxide (GO) was dispersed in $\mathrm{KOH}$ aqueous solution to expand the layers, and the GO was hydrothermalized to remove moisture. ${ }^{19}$ Although the proposed storage material here (KGO) is somewhat similar to other porous materials, the storage mechanism is very different. In our storage mechanism, the $\mathrm{H}_{2}$ molecules vigorously move around inside the potential well, which originated mainly from the $\mathrm{H}_{2}$-graphene layer interaction. This is clearly distinct from the $\mathrm{H}_{2}$ storage mechanism in the aforementioned study, where a hydrogen molecule binds at a particular binding site and the storage material (whether it is a MOF, any carbon-based material, or otherwise) is used to provide the scaffold structure to maintain the binding sites. In the material we propose, however, a more or less uniform potential in the void space is desirable to achieve gas-phase storage in the potential well.

\section{Methods}

We performed $a b$ initio density functional theory (DFT) electronic structure and total energy calculations ${ }^{\mathbf{2 0 , 2 1}}$ and obtained the $\mathrm{H}_{2}$ potential energy surface (PES) of the system. In the calculations, the generalized gradient approximation of Perdew et al. ${ }^{22}$ with Grimme-type ${ }^{23}$ van der Waals interaction correction was employed to describe the exchange-correlation energy in the Vienna ab initio simulation package (VASP). ${ }^{24}$ An energy cutoff of $400 \mathrm{eV}$ was used for the projector-augmented wave pseudopotential basis set. In the atomic structure relaxation, the atomic configuration was adjusted until the HellmannFeynman force on each atom was less than $0.01 \mathrm{eV} \AA^{-1}$. To quantify the storage capacity of the materials at finite temperatures, we performed grand canonical Monte Carlo (GCMC) simulations. In the GCMC simulations, a hydrogen molecule was modelled as a single classical point particle, and the intermolecular $\left(\mathrm{H}_{2}-\mathrm{H}_{2}\right)$ interaction was described by the Silvera-Goldman potential. ${ }^{25}$

\section{Results and discussion}

Local atomic configurations and the chemical composition of KGO depend strongly on the synthesis conditions, ${ }^{\mathbf{2 6}}$ and their precise determination in experiments is still difficult. On the theoretical side, there were efforts to search for structures of GO with various functional group coverage ${ }^{27,28}$ but only a small set of configurations were examined in most cases due to computational limitations. After the calculation of atomic relaxations, we found that all oxygen atoms were strongly bound to carbon atoms and formed epoxy groups. Potassium atoms were attached near the oxygen atoms (Fig. 1). For computational simplicity, pillar molecules between the layers (which should exist in reality to maintain the interlayer distance, $d$ ) were ignored, and $d$ was kept constant in the relaxation calculation.

We define the potential energy of a $\mathrm{H}_{2}$ molecule as a function of its position in the space between layers as follows:

$$
V(\vec{r})=E\left[\mathrm{M}+\mathrm{H}_{2}(\vec{r})\right]-E[\mathrm{M}]-E\left[\mathrm{H}_{2}\right]
$$

where $\mathrm{M}$ is the storage material (KGO) interacting with $\mathrm{H}_{2}$, and $E[\mathrm{~S}]$ is the total energy of the system, where $\mathrm{S}$ stands for the materials constituting the system.

Fig. 2 shows the PES of KGO (the atomic configuration is displayed in the inset and its chemical formula is $\mathrm{CO}_{0.25} \mathrm{~K}_{0.125}$, unless stated otherwise) with $d=10 \AA$ in the plane parallel $(x-y)$ to the graphene layer at $z=5 \AA$ (green parallelogram in the inset). In the calculation, the $x-y$ plane is partitioned into $30 \times 30$ grids parallel to the lattice vector of a unit supercell, and energy optimization is done with the constraint of the centers of the $\mathrm{H}_{2}$ molecules kept fixed at each grid point. The PES of the arbitrary points is obtained by spline interpolation. The regions of positive

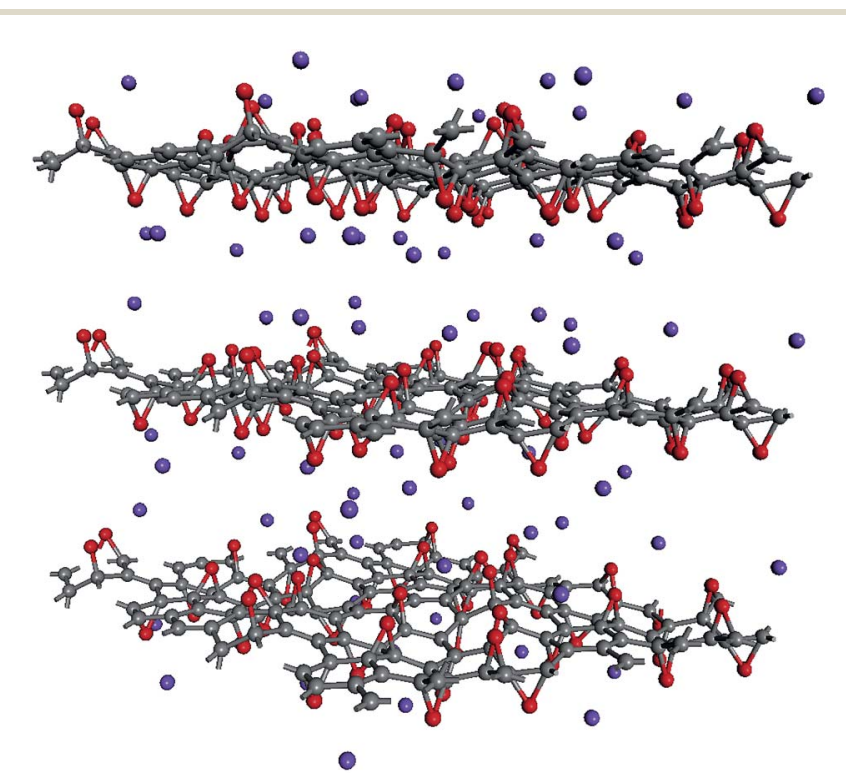

Fig. 1 A model of the atomic structure of KGO. Gray, red and purple dots represent carbon, oxygen and potassium atoms, respectively. 


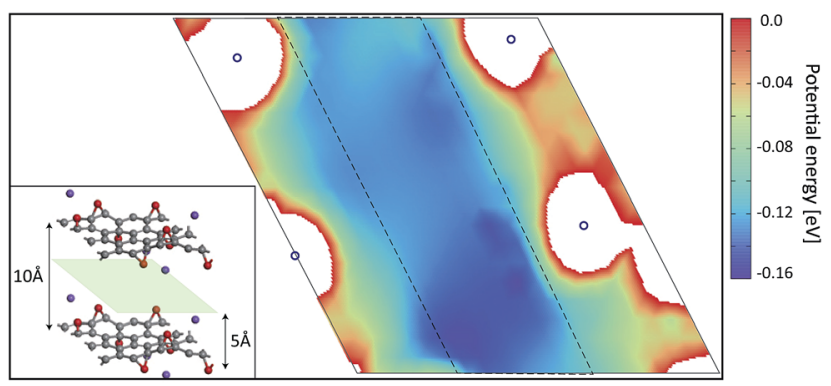

Fig. 2 The potential energy surface of KGO with $d=10 \AA$ in the plane passing through the center of two graphene layers (the light green parallelogram in the inset). The region of positive potential energy is shown in white color. The positions of 4 potassium atoms are denoted by blue circles. The inset shows the atomic configuration of the unit supercell in the ab initio calculation

potential energy (thus inaccessible to the $\mathrm{H}_{2}$ molecules) are represented in white color. It is clearly seen from Fig. 2 that the potential energy is relatively uniform in the plane, except in the regions close to the oxygen and potassium atoms. The enhancement and uniformity of the attractive interaction are especially remarkable in the region denoted by a dashed parallelogram in Fig. 2. The average depth of the attractive potential well in this region reaches $-0.12 \mathrm{eV}$, and this region provides an efficient hydrogen diffusion pathway.

In order to understand the reason for the potential strength enhancement by the epoxy groups and intercalated potassium ions, we also calculated the PES of the simplest building block of KGO, i.e. a single potassium-attached epoxy (K-epoxy) group on the $4 \times 4$ graphene supercell. Fig. 3 shows the PES in the plane at $z=3 \AA$ (the light parallelogram in the inset). The average potential energy of the accessible region (where $V<0$ ) at the plane $3 \AA$ above the graphene layer is $-83 \mathrm{meV}$, which is 23 $\mathrm{meV}$ stronger than that of the pristine graphene layer $(-60 \mathrm{meV}$, via van der Waals interaction only). The deepest potential well near the potassium ion reaches $-0.16 \mathrm{eV}$. The enhancement of the attractive potential by functionalization is attributed to the induced dipole interactions of $\mathrm{H}_{2}$ with the electric field

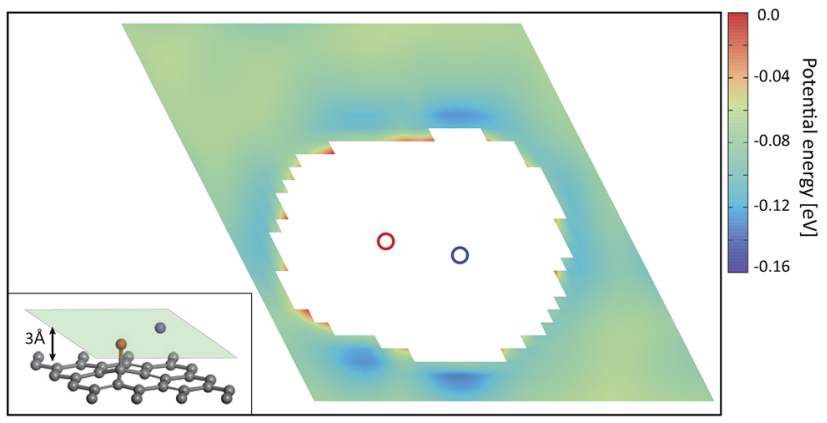

Fig. 3 The potential energy surface of a $4 \times 4$ graphene supercell with a potassium-attached epoxy group. The plane of the plot is parallel with and $3 \AA$ above the graphene layer (the light green parallelogram in the inset). The region of positive potential energy is shown in white color. The positions of the oxygen and potassium atoms are denoted by red and blue circles, respectively. The inset shows the atomic configuration of the unit supercell in the ab initio calculation. generated by oxygen atoms or potassium ions (and minute orbital hybridization). ${ }^{10,29}$ According to atomic charge analysis, ${ }^{30}$ a potassium atom donates nearly one electron to graphene and the epoxy group, and becomes a positive ion $\left(\mathrm{K}^{+}\right)$. The magnitude of the electric field at the position of the largest potential is $1.78 \mathrm{~V}^{-1}$, and we can estimate roughly that the largest contribution of the induced dipole interaction to the binding energy $\left(E_{\text {ind }}=-\frac{1}{2} \alpha|\vec{E}|^{2}\right.$, where $\alpha$ is the polarizability of the $\mathrm{H}_{2}$ molecule) is $-0.11 \mathrm{eV}$ (the average contribution is a few tens of $\mathrm{meV})$.

Using the PES obtained by $a b$ initio calculations, we modelled the effective potentials used in the GCMC simulation. The interaction between a hydrogen molecule and KGO is assumed to be the sum of the potentials contributed to by each constituent of KGO, i.e. the graphene layer, the epoxy group or the potassium-attached epoxy group (K-epoxy):

$$
\begin{aligned}
V_{\mathrm{H}_{2}-\mathrm{KGO}}(\vec{r})= & V_{\mathrm{H}_{2}-\mathrm{G}}(\vec{r})+\sum_{i} V_{\mathrm{H}_{2}-\mathrm{O}}\left(\vec{r}-\vec{r}_{\mathrm{O}, i}\right) \\
& +\sum_{i} V_{\mathrm{H}_{2}-\mathrm{KO}}\left(\vec{r}-\vec{r}_{\mathrm{KO}, i}\right)
\end{aligned}
$$

where $V_{\mathrm{H}_{2}-\mathrm{G}}(\vec{r})$ is the interaction potential between $\mathrm{H}_{2}$ and the graphene layers, $V_{\mathrm{H}_{2}-\mathrm{O}}\left(\vec{r}-\vec{r}_{\mathrm{O}, i}\right)$ is the potential between $\mathrm{H}_{2}$ and the $i^{\text {th }}$ epoxy group, and $V_{\mathrm{H}_{2}-\mathrm{KO}}\left(\vec{r}-\vec{r}_{\mathrm{KO}, i}\right)$ is the potential between $\mathrm{H}_{2}$ and the $i^{\text {th }} \mathrm{K}$-epoxy group. The interactions between $\mathrm{H}_{2}$ and the epoxy/K-epoxy/carbon are described by the LennardJones potential, $\left(V(r)=4 \varepsilon\left(\left(\frac{\sigma}{r}\right)^{12}-\left(\frac{\sigma}{r}\right)^{6}\right)\right)$, where $r^{\text {cutoff }}$ is the cutoff distance. In minimizing the total (integrated) deviation of the Lennard-Jones potentials from the ab initio potentials, we used the local weighting factor, $\exp \left[-V_{\mathrm{ab}}(\vec{r}) / k_{\mathrm{B}} T\right]$, because reproducing low potential regions is more important than reproducing high potential regions. The parameters obtained from this procedure are listed in Table 1. The PES on the cross section between two adjacent KGO layers, obtained from $a b$ initio calculations, is shown in Fig. 4(a), and the one obtained from the parameterized Lennard-Jones potentials is shown in Fig. 4(b), which reproduces Fig. 4(a) reasonably well (within $0.03 \mathrm{eV}$ deviation in most regions).

The GCMC simulation results of the gravimetric storage capacity as a function of the pressure $(P \leq 10 \mathrm{MPa}$ at $300 \mathrm{~K})$ of KGO with different $d$ values are shown in Fig. 5 . The isotherms of KGO with three different $d$ values $(d=8,9$ and $10 \AA)$ show similar behavior, but it was noticed that when $d=10 \AA$, the highest storage capacity is shown among the three different $d$ values as the external pressure increases. The gravimetric storage density reaches $2.5 \mathrm{wt} \%$ at $10 \mathrm{MPa}$. The storage capacity of KGO with $d=$

Table 1 The parameters of the Lennard-Jones interaction potential, $\varepsilon_{\mathrm{H}_{2}-\mathrm{A}}$ and $\sigma_{\mathrm{H}_{2}-\mathrm{A}}$, and the interaction cutoff, $r_{\mathrm{H}_{2}-\mathrm{A}}^{\text {cutoff }}$, for $\mathrm{A}=$ epoxy, $\mathrm{K}-$ epoxy, and carbon

\begin{tabular}{llll}
\hline A & $\varepsilon_{\mathrm{H}_{2}-\mathrm{A}}[\mathrm{meV}]$ & $\sigma_{\mathrm{H}_{2}-\mathrm{A}}[\AA]$ & $r_{\mathrm{H}_{2}-\mathrm{A}}^{\text {cutoff }}[\AA]$ \\
\hline Carbon & 5.4 & 2.97 & 6.0 \\
Epoxy & 11.0 & 2.70 & 7.0 \\
K-Epoxy & 60.0 & 2.50 & 7.0
\end{tabular}


(a)

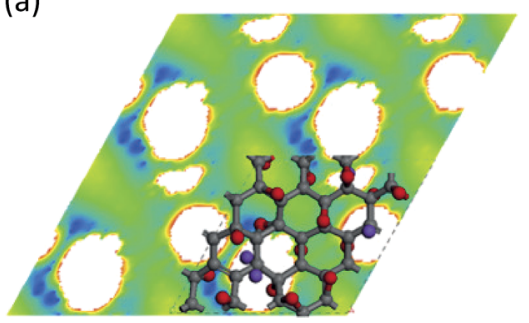

(b)

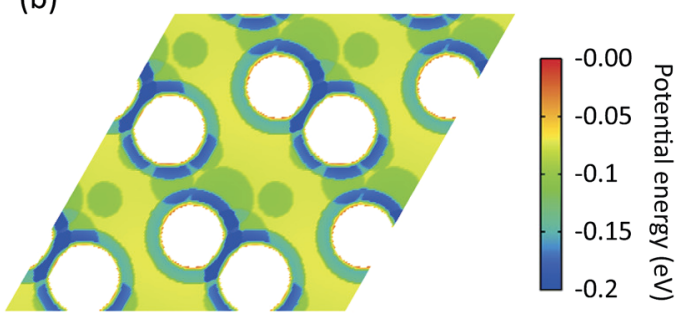

Fig. 4 (a) The potential energy experienced by $\mathrm{H}_{2}$ in the plane passing through the center between two KGO layers separated by $9 \AA$, using ab initio calculations. (b) The same potential energy surface obtained from Lennard-Jones potentials optimally fitted to the ab initio calculations in (a).

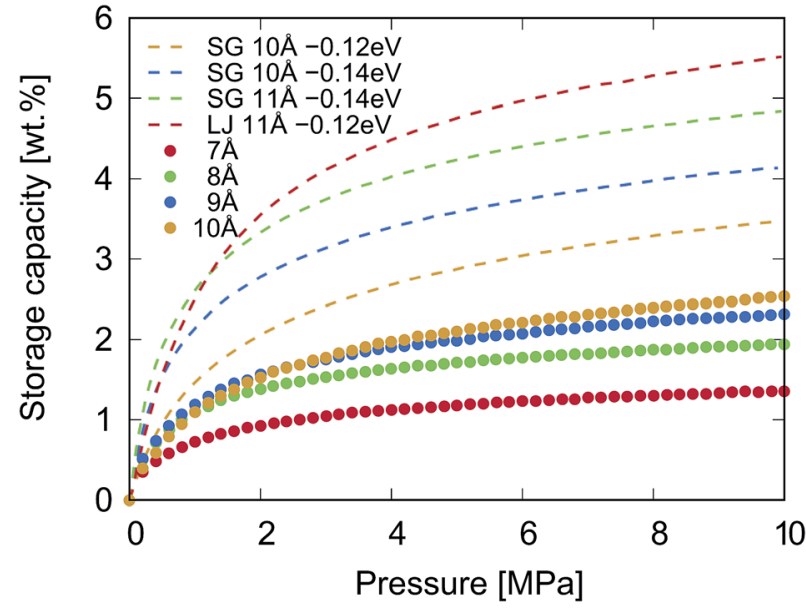

Fig. 5 The dotted curves represent the calculated gravimetric storage capacity of $\mathrm{KGO}\left(\mathrm{CO}_{0.25} \mathrm{~K}_{0.125}\right)$ with different $d$ values at $300 \mathrm{~K}$. The interaction between $\mathrm{H}_{2}$ and $\mathrm{KGO}$ was described by Lennard-Jones potentials, and that between $\mathrm{H}_{2}$ molecules was described by the Silvera-Goldman potential. The dashed curves represent the structural model with a given uniform potential, $V_{\mathrm{EFF}}$, as explained in the text. The interaction between $\mathrm{H}_{2}$ molecules was due to either the SilveraGoldman potential (SG) or the Lennard-Jones potential (LJ). The dashed curves, representing the capacity with uniform potential, were reprinted with permission from ref. 19, copyright 2016, Elsevier.

$7 \AA$ is smaller than that of the others in the whole range of pressure. The trend in storage capacity can be explained in terms of the density enhancement factor and the accessible volume, $V_{\text {acc }}$ (with negative potential energy), inside the KGO layers. The interlayer distance is one of the key factors in determining these two values by changing the $\mathrm{H}_{2}$ PES and the volume of the void space inside KGO. Since the accessible volume is of major importance in high pressure storage, increasing the interlayer distance results in the increase of storage capacity at $10 \mathrm{MPa}$. However, as far as the present structure of KGO is concerned, if we increase $d$ further, beyond $10 \AA$, the capacity ceases to increase because the dispersion interaction decreases when the interlayer distance becomes too large. With the present atomic structure of $\mathrm{KGO}$, the gravitational storage density at room temperature reaches $2.5 \mathrm{wt} \%$ at $10 \mathrm{MPa}$. We will explain later that the storage capacity can increase up to $5.5 \mathrm{wt} \%$ with a different choice of uniform average potential, $V_{\mathrm{EFF}}$. In the following discussion, we will explain how the PES and the accessible volume determine the storage capacity in detail.

The interaction between hydrogen and KGO gives rise to the formation of the relatively uniform attractive potential well between KGO layers, and the equilibrium hydrogen density inside KGO, $n_{\text {in }}$, is much higher than that outside KGO (the $\mathrm{H}_{2}$ reservoir), $n_{\text {out }}$. For an ideal gas, the local equilibrium density enhancement at position $\vec{r}$ follows the Boltzmann factor, $n_{\text {in }}(\vec{r})=n_{\text {out }} \mathrm{e}^{-\frac{V(\vec{r})}{k_{\mathrm{B}} T}}$, as mentioned before. The average density enhancement factor, $X=\frac{n_{\text {in }}}{n_{\text {out }}}$, is given by $\frac{1}{V_{\text {acc }}} \int_{V_{\text {acc }}} \mathrm{e}^{\frac{-V(\vec{r})}{k_{\mathrm{B}} T}} \mathrm{~d} \vec{r}$. In the case of a uniform potential, $V(\vec{r})=V_{0}$, and $X$ is given by $\mathrm{e}^{\frac{-V_{0}}{k_{\mathrm{B}} T}}$ as stated in the introduction. For a non-ideal gas, however, the contribution of intermolecular interactions to the chemical potential inside the material $\left(\mu_{\text {int }}\left(n_{\text {in }}\right)\right)$ is not negligible and should be taken into account to obtain the correct density enhancement. $n_{\text {in }}$ is determined by solving the chemical potential equality inside and outside KGO:

$$
\mu_{\mathrm{kin}}\left(n_{\mathrm{in}}\right)+V_{\mathrm{EFF}}+\mu_{\text {int }}\left(n_{\text {in }}\right)=\mu\left(n_{\text {out }}\right) .
$$

Outside the storage material, the $\mathrm{H}_{2}-\mathrm{H}_{2}$ interaction is sufficiently small as long as we are interested in the pressure, $P \leq$ $10 \mathrm{MPa}$, at room temperature. We make an ideal gas approximation that $\mu\left(n_{\text {out }}\right)=k_{\mathrm{B}} T \log \left(n_{\text {out }} \lambda^{3}\right)$, where $\lambda$ is the thermal wavelength $\left(\lambda=\frac{h}{\sqrt{2 \pi m k_{\mathrm{B}} T}}\right.$, where $h$ is the Planck constant and $m$ is the mass of the $\mathrm{H}_{2}$ molecule). The contribution to $\mu$ from rotational and vibrational motion is not included here and will be discussed later. In eqn (3), the kinetic energy contribution to $\mu$ inside the storage material has the same expression as outside, $\mu_{\text {kin }}\left(n_{\text {in }}\right)=k_{\mathrm{B}} T \log \left(n_{\mathrm{in}} \lambda^{3}\right)$. We separate the chemical potential contribution from the external potential and intermolecular interaction into two parts $\left(V_{\mathrm{EFF}}+\mu_{\text {int }}\left(n_{\text {in }}\right)\right)$ : $V_{\mathrm{EFF}}=-k_{\mathrm{B}} T \log \left(\frac{1}{V_{\mathrm{acc}}} \int_{V_{\mathrm{acc}}} \exp \left[-\frac{V(\vec{r})}{k_{\mathrm{B}} T}\right] \mathrm{d}^{3} \vec{r}\right)$ is the effective external potential for an ideal gas, and $\mu_{\text {int }}(n)=-k_{\mathrm{B}} T \log$ $\left(\frac{1}{V_{\text {acc }} N} \int_{V_{\text {acc }^{N}}}\left\langle\exp \left[-\frac{V\left(\vec{r} ; \vec{r}^{N-1}\right)+V_{\text {int }}\left(\vec{r} ; \vec{r}^{N-1}\right)}{k_{\mathrm{B}} T}\right]\right\rangle_{N-1} \mathrm{~d}^{3 N} \vec{r}^{N}\right)-V_{\mathrm{EFF}} \quad$ is 
the chemical potential from intermolecular interaction. ${ }^{16,31} N$ is the number of particles in volume $V_{\text {acc }}\left(N=n V_{\text {acc }}\right),\langle\ldots\rangle_{N}$ denotes the canonical ensemble average over the configuration space of the $N$ - 1 particle system and $V_{\text {(int) }}\left(\vec{r} ; \vec{r}^{N-1}\right)$ is the external (interaction) potential between a particle at $\vec{r}$ and other $N-1$ particles at $N-1$ particle configuration space, $\vec{r}^{N-1}=\left(\vec{r}_{1}, \vec{r}_{2}, \ldots, \vec{r}_{N-1}\right)$. Silvera-Goldman potentials are used for $V_{\text {int }}\left(\vec{r} ; \vec{r}^{N-1}\right) .{ }^{25}$ If we were to adopt a more attractive $\mathrm{H}_{2}-\mathrm{H}_{2}$ interaction than Silvera-Goldman, the enhancement factor and the corresponding storage capacity would further increase. Fig. 6 shows a graphical solution of eqn (3) and the density enhancement as a function of the $\mathrm{H}_{2}$ pressure in the outside reservoir. For example, if the $\mathrm{H}_{2}$ pressure outside KGO is $5 \mathrm{MPa}$ (the green dot in Fig. 6(a)), the chemical potential is $\approx-0.2 \mathrm{eV}$. The corresponding $\mathrm{H}_{2}$ density inside the KGO layer (the solution of eqn (3)) is $\approx 0.03 \AA^{-3}$ (the red dot in Fig. 6(a)) and the density enhancement factor, $X$, at room temperature $(T=300 \mathrm{~K})$ is $\approx 24$. Not all of the space inside the KGO layer is available for storage, because the strong repulsive interaction between $\mathrm{H}_{2}$ molecules and the functional groups prohibits hydrogen molecules from approaching the region of positive potential near the functional groups. Storage capacity is essentially the product of three quantities: the density in the outside KGO layer, the volume of the space of negative potential inside the KGO layer, and the density enhancement factor due to the negative potential, i.e. the storage capacity $=c n_{\text {out }} X V_{\text {acc. }}$ The proportionality constant, $c$, is related to the mass density of KGO.

At a very low pressure ( $\lesssim 1 \mathrm{MPa})$, the hydrogen density both inside and outside $\mathrm{KGO}$ is so low that the average intermolecular distance is larger than the $\mathrm{H}_{2}-\mathrm{H}_{2}$ interaction range. In this case, the hydrogen molecules behave like an ideal gas and the contribution of intermolecular interactions to the chemical potential is very small. The chemical potential difference between the interacting gas (solid red curve) and the ideal gas inside (dashed red curve) the KGO layer is very small (Fig. 6(a)), and the corresponding density enhancement factor is approximately given by $\mathrm{e}^{-\frac{V_{\mathrm{EFF}}}{k_{\mathrm{B}} T}}$ (Fig. 6(b)). Thus the storage capacity is linearly related to pressure, and its slope is proportional to $\frac{1}{k_{\mathrm{B}} T} \mathrm{e}^{-\frac{V_{\mathrm{EFF}}}{k_{\mathrm{B}} T}} V_{\text {acc }}$. Based on this argument, we will explain the isotherms in Fig. 5. The initial slope of the isotherm for KGO with $d=8 \AA$ is the steepest among the four cases $(d=7,8,9$ and $10 \AA$ ). This indicates that $\left|V_{\mathrm{EFF}}\right|$ is largest when $d=8 \AA$, because $8 \AA$ is about twice the equilibrium distance between the hydrogen molecule and a single layer of KGO; the hydrogen molecules interact with both the upper and lower layers. The magnitude of $V_{\mathrm{EFF}}$ for KGO with $d=7 \AA$ is similar to that for KGO with $d=8 \AA$, but $V_{\text {acc }}$ for $d=7 \AA$ is smaller than $V_{\text {acc }}$ for $d=$ $8 \AA$, so the storage capacity for $d=7 \AA$ is smaller than that for $d=8 \AA$. Although $V_{\text {acc }}$ for $d=8 \AA$ is less than that for $d=9 \AA$ or $d=10 \AA$, the storage capacity depends linearly on $V_{\text {acc }}$ but exponentially on $V_{\mathrm{EFF}}$, so that increasing $\left|V_{\mathrm{EFF}}\right|$ is more effective for low pressure storage.

At a higher external pressure, however, the contribution of the repulsive intermolecular interaction to the chemical potential is substantial. In this case, the total effective potential $\left(V_{\mathrm{EFF}}+\mu_{\mathrm{int}}\right)$ becomes less attractive, and the density enhancement factor gets smaller. Here, a larger $V_{\text {acc }}$ becomes a more important factor than in the lower pressure case, and a larger $d$ creates a larger $V_{\text {acc }}$ inside the layers, leading to a higher storage capacity. Fig. 7(a) presents a snapshot of the GCMC simulation for KGO with $d=10 \AA$. A quasi-2D bilayer structure of the distribution of hydrogen molecules (indicated by two arrows in Fig. 7(b)) is formed in $d=10 \AA$ KGO layers, and the distance between the two hydrogen molecule layers is in the range of the attractive $\mathrm{H}_{2}-\mathrm{H}_{2}$ interaction.

In the dense hydrogen environment, an epoxy group may be reduced to a hydroxyl group. ${ }^{32,33}$ The binding energy (or PES) between a hydrogen molecule and a hydroxyl group is similar to that between a hydrogen molecule and an epoxy group. Because of the strong bond between the epoxy group and a $\mathrm{K}$ ion $(2.5 \mathrm{eV})$, the migration of a $\mathrm{K}$ ion from the epoxy group to the hydroxyl group is unfavorable and the $\mathrm{K}$ ions remained near the epoxy

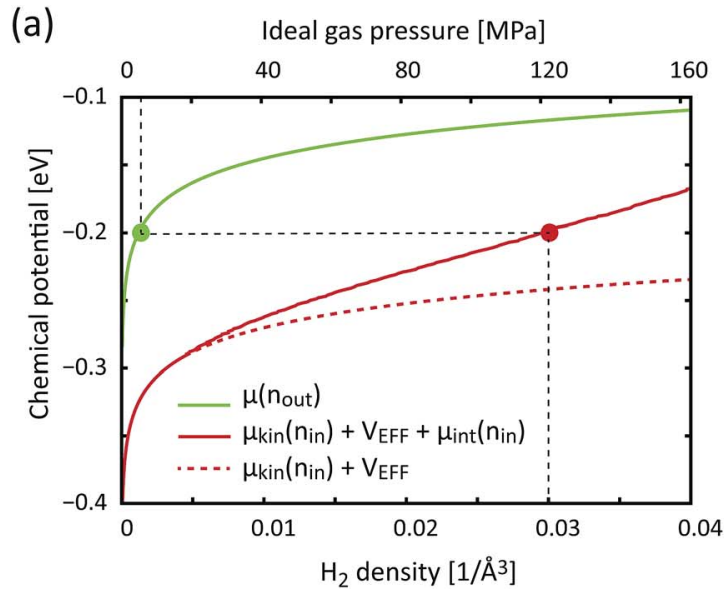

(b)

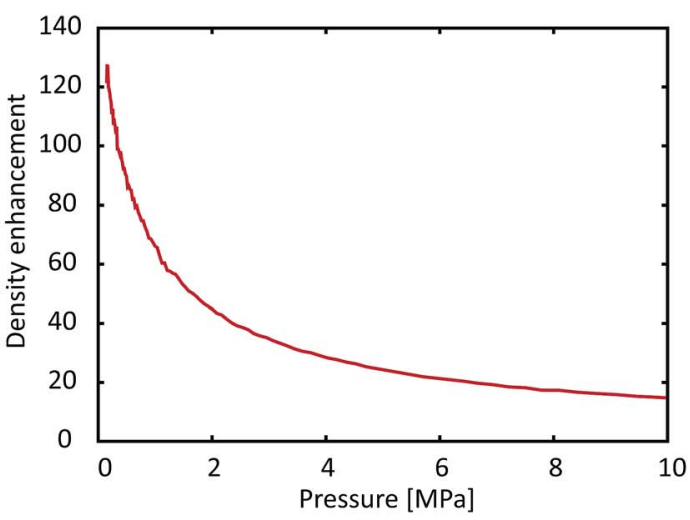

Fig. 6 (a) The chemical potential as a function of the $\mathrm{H}_{2}$ density (lower $x$-axis), outside (green curve, RHS of eqn (3)) and inside (solid red curve, LHS of eqn (3)) KGO, with $d=10 \AA$. . For comparison, the dashed red curve represents the chemical potential inside the KGO layer under the ideal gas assumption. The upper $x$-axis is the ideal gas pressure corresponding to the gas density on the lower $x$-axis. (b) The density enhancement factor, $X$, as a function of the $\mathrm{H}_{2}$ pressure outside the $\mathrm{KGO}$ layer. $T=300 \mathrm{~K}$ in all cases. 
(a)

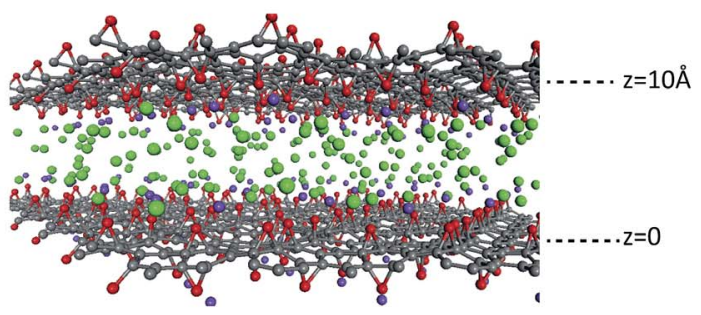

(b)

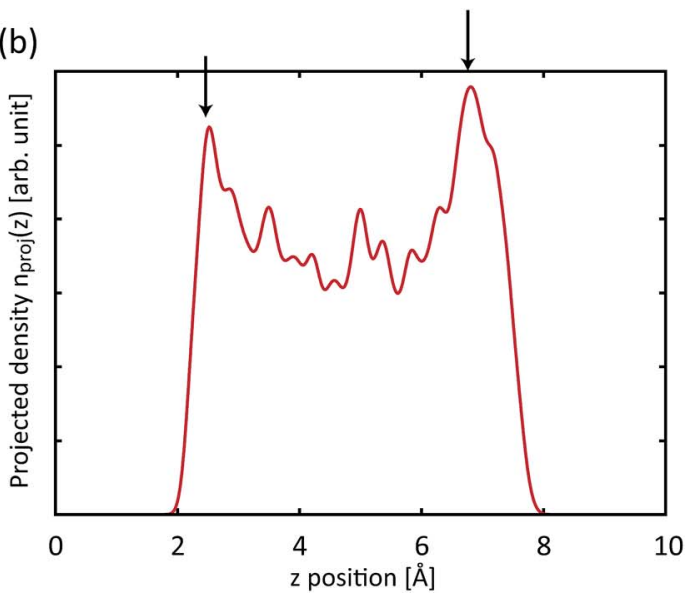

Fig. 7 (a) A snapshot of the grand canonical Monte Carlo simulation after reaching equilibrium. Gray, red, purple and green dots represent carbon, oxygen, potassium and hydrogen molecules, respectively. (b) The $z$-axis-projected density of hydrogen molecules, $n_{\text {proj }}(z)=\int_{x y} n(x, y, z) \mathrm{d} x \mathrm{~d} y$, between two KGO layers located at $z=0$ and $z=10 \AA$. Peak positions in the distribution of hydrogen molecules are indicated by arrows.

groups, so the interaction between the hydrogen molecule and the storage material is hardly changed by the reduction.

We then decided to examine another aspect of the storage material, namely the influence of the coverage of functional groups on the storage capacity. We calculated the hydrogen storage capacity of KGO with $d=10 \AA$ for different functional group densities. Fig. 8 shows the computational results with different functional group coverages: $\mathrm{C}_{32} \mathrm{O}_{4} \mathrm{~K}_{2}, \mathrm{C}_{32} \mathrm{O}_{8} \mathrm{~K}_{4}$, $\mathrm{C}_{32} \mathrm{O}_{12} \mathrm{~K}_{6}$ and $\mathrm{C}_{32} \mathrm{O}_{16} \mathrm{~K}_{8}$. Since the epoxy and $\mathrm{K}$-epoxy groups increase the magnitude of $V_{\mathrm{EFF}}$ (Fig. 3), the gravimetric storage capacity increases as the functional group density increases in the low pressure range $(P \leq 2 \mathrm{MPa})$. However, the isotherms of KGO at higher pressures show that the storage capacity cannot be increased further by too high a functional group coverage. The increase in the weight of the functional groups and the expansion of the volume of the steric hindrance region are

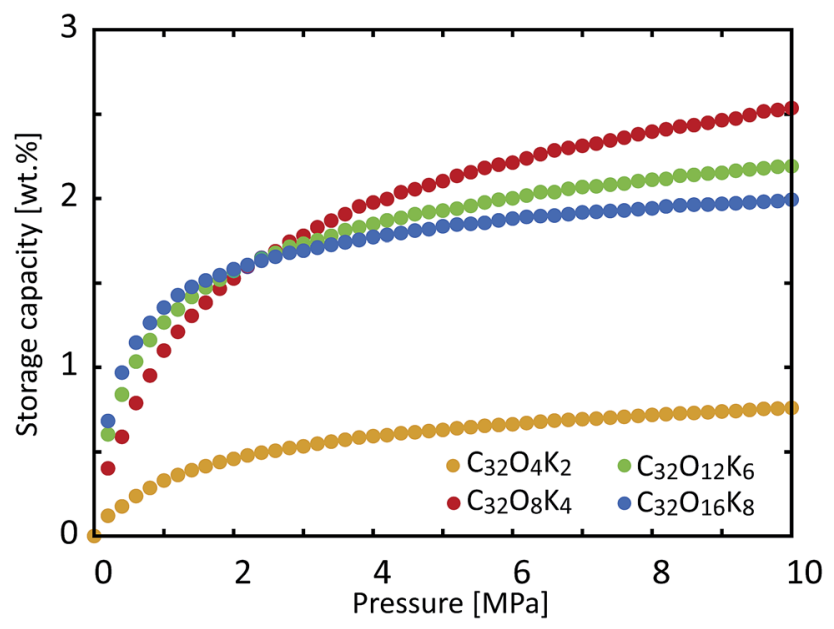

Fig. 8 Room temperature storage capacity of $\mathrm{KGO}$ with $d=10 \AA$ for different functional group coverages: $\mathrm{C}_{32} \mathrm{O}_{4} \mathrm{~K}_{2}, \mathrm{C}_{32} \mathrm{O}_{8} \mathrm{~K}_{4}, \mathrm{C}_{32} \mathrm{O}_{12} \mathrm{~K}_{6}$ and $\mathrm{C}_{32} \mathrm{O}_{16} \mathrm{~K}_{8}$. responsible for the decrease in storage capacity. For the reasons mentioned above, we found that the $\mathrm{C}_{32} \mathrm{O}_{8} \mathrm{~K}_{4}$ case (whose coverage is $1 \mathrm{~K}$ atom per 4 unit cells of graphene, or $1 \mathrm{~K}$ atom per $20.96 \AA^{2}$ ) is the optimal condition for hydrogen storage, and its storage capacity reaches $2.5 \mathrm{wt} \%$ at $10 \mathrm{MPa}$ and room temperature.

In the GCMC simulations, we calculated the thermodynamic quantities under the classical point particle approximation. In this approximation, we replaced the quantum mechanical partition function $\left(Q=\sum_{n} \mathrm{e}^{-\frac{E_{n}}{k_{\mathrm{B}} T}}\right)$ with phase-space integration $\left(Q=\int \exp \left[-\frac{H\left(\vec{r}^{N}, \vec{p}^{N}\right)}{k_{\mathrm{B}} T}\right] \mathrm{d}^{N} \vec{r} \mathrm{~d}^{N} \vec{p}\right.$, where $\left(\vec{r}^{N}, \vec{p}^{N}\right)$ represents the position and momentum coordinates in the $N$-particle phase-space) and ignored the vibrational and rotational degrees of freedom of the diatomic molecule. The quantum mechanical correction due to confinement in the $z$-direction was considered by directly solving the single particle Schrödinger equation in the $z$-direction. The PES of the hydrogen molecule inside KGO with $d=10 \AA$ (Fig. 2) was used as an example of our quantitative analysis. Our calculation of the partition function for the quantum point particle turned out to be slightly lower $(\approx 3 \%)$ than that of the classical point particle, indicating that the classical single particle approximations slightly overestimate the storage capacity. Although the vibrational modes are not excited at room temperature due to the large vibrational energy $(h \nu)$ compared to $k_{\mathrm{B}} T$, the zero point energy $\left(\frac{1}{2} h \nu\right)$ can contribute to the partition function. The calculated vibrational energy of the $\mathrm{H}_{2}$ molecule at the energy minimum position of KGO with $d=10 \AA$ (Fig. 2) is $527.5 \mathrm{meV}$, which is $5.5 \mathrm{meV}$ lower than that of a free $\mathrm{H}_{2}$ molecule. The softening of the $\mathrm{H}_{2}$ vibration mode $\left(\Delta E_{\mathrm{vib}}<0\right)$ leads to an increase in the partition 
function inside $\mathrm{KGO}$ by $\mathrm{e}^{-\frac{\Delta E_{\mathrm{vib}}}{2 k_{\mathrm{B}} T}}-1 \approx 11 \%$ at room temperature..$^{17,34}$

In the definition of the PES (eqn (1)), we assumed that the direction of the $\mathrm{H}_{2}$ molecule is fully relaxed to the minimum energy configuration, i.e. $E\left[\mathrm{M}+\mathrm{H}_{2}(\vec{r})\right] \equiv \operatorname{Min}_{\hat{\Omega}} E\left[\mathrm{M}+\mathrm{H}_{2}(\vec{r}, \hat{\Omega})\right]$ (where $\hat{\Omega}(\theta, \varphi)$ is the direction of the $\mathrm{H}_{2}$ molecule and $E[\mathrm{M}+$ $\left.\mathrm{H}_{2}(\vec{r}, \hat{\Omega})\right]$ is the total energy of the storage material $\mathrm{M}$ and $\mathrm{H}_{2}$ molecules). In order to account for the decrease of the partition function due to rotational motion of the $\mathrm{H}_{2}$ molecule with respect to the direction of the minimum energy, we computed the angle $(\hat{\Omega})$ dependent energy, $E\left[\mathrm{M}+\mathrm{H}_{2}(\vec{r}, \hat{\Omega})\right]$, at position $\vec{r}_{0}$ near the energy minimum position of the same system used in the vibrational motion analysis above, and obtained the angle averaged potential energy,

$$
V\left(\vec{r}_{0}\right)_{\hat{\Omega}}=\frac{\int E\left[\mathrm{M}+\mathrm{H}_{2}\left(\vec{r}_{0}, \hat{\Omega}\right)\right] \exp \left(-\frac{E\left[\mathrm{M}+\mathrm{H}_{2}\left(\vec{r}_{0}, \hat{\Omega}\right)\right]}{k_{\mathrm{B}} T}\right) \mathrm{d} \hat{\Omega}}{\int \exp \left(-\frac{E\left[\mathrm{M}+\mathrm{H}_{2}\left(\vec{r}_{0}, \hat{\Omega}\right)\right]}{k_{\mathrm{B}} T}\right) \mathrm{d} \hat{\Omega}}
$$

The difference between the angle averaged potential and the minimum potential with respect to the rotation angle, $\Delta V\left(\vec{r}_{0}\right)_{\text {rot }}$ $\left(=V\left(\vec{r}_{0}\right) \hat{\Omega}-V\left(\vec{r}_{0}\right)\right)$, is $2.6 \mathrm{meV}$, so the rotational motion of the $\mathrm{H}_{2}$ molecule reduces the partition function inside KGO by $\mathrm{e}^{\frac{-\Delta V\left(\vec{r}_{0}\right)_{\text {rot }}}{k_{\mathrm{B}} T}}-1 \approx-10 \%$. The net correction to the partition function from the above three contributions is $\approx-3 \%(=0.97 \times$ $1.11 \times 0.9$ ), and this means that the single particle assumption of the $\mathrm{H}_{2}$ molecule in the classical picture is still valid in the context of the storage capacity calculation.

Previously, people attempted to decorate storage materials with various TM atoms, but the clustering of TM atoms posed a serious synthesis problem. ${ }^{35,36}$ The present system is free from metal atom clustering; the $\mathrm{K}$ ions are prevented from clustering by the repulsive electrostatic interaction between the positively charged potassium ions. Our calculations show that the binding energy between the potassium atom and the epoxy group (2.5 $\mathrm{eV})$ is larger than the cohesive energy of potassium $(1.0 \mathrm{eV})$, and the configuration of separated potassium atoms is more stable than that of aggregated ones.

So far, we have investigated in detail the hydrogen storage capacity of a particular structure of KGO. The calculated roomtemperature storage capacity of $2.5 \mathrm{wt} \%$ at $10 \mathrm{MPa}$ is better than most storage materials using the physisorption of $\mathrm{H}_{2}$ molecules, but is still not satisfactory for practical applications. It is noteworthy that the storage capacity depends on the atomic constituents and structures. To make more general predictions (possibly with different potential strengths) of the storage capacity and determine the range of the storage capacity using the present mechanism (storage in the potential well in the $\mathrm{H}_{2}$ gas form), we also performed GCMC simulations using different potential strengths between $\mathrm{H}_{2}$ and the scaffold materials, $V_{\mathrm{EFF}}$. The storage capacity for a given uniform average potential strength, $V_{\mathrm{EFF}}$, is juxtaposed with the results presented before as dashed curves in Fig. 5. For the cases of $V_{\mathrm{EFF}}=-0.12$ and $-0.14 \mathrm{eV}$ with $d=10 \AA$, the gravimetric density at $10 \mathrm{MPa}$ and $300 \mathrm{~K}$ reaches about 3.5 and $4.2 \mathrm{wt} \%$ respectively with the
Silvera-Goldman $\mathrm{H}_{2}-\mathrm{H}_{2}$ interaction. If $d$ becomes $11 \AA$ with $V_{\mathrm{EFF}}=-0.14 \mathrm{eV}$, the capacity further increases to $4.8 \mathrm{wt} \%$. There also exists a dependence of storage capacity on the choice of $\mathrm{H}_{2}-\mathrm{H}_{2}$ interaction: the Lennard-Jones potential gives about $5.5 \mathrm{wt} \%$ for $d=11 \AA$ with $V_{\mathrm{EFF}}=-0.12 \mathrm{eV}$. Some of the results (dashed curves) were previously reported in the literature. ${ }^{19}$ These results indicate that there is significant room for enhancement of the storage capacity using other atoms or molecules for intercalation, which can provide different $V_{\mathrm{EFF}}$ or $d$ values. We note that the storage capacity increases rapidly with potential strength, since the capacity essentially depends on it exponentially. One should be cautioned, however, that such a sensitive dependence sets a certain limit on the accuracy of the calculated storage capacity.

\section{Conclusions}

In summary, we proposed a novel hydrogen storage mechanism in the potential well inside the void space of porous materials, and showed that potassium-intercalated graphite oxide can provide such a void space with a negative potential to realize room temperature hydrogen storage. A relatively uniform potential well was found to be created between the layers and the origin of the enhanced attractive potential was closely examined. The dependence of the hydrogen storage capacity on the interlayer distance and the coverage of functional groups was explained in terms of the effective external potential and the corresponding density enhancement factor.

\section{Acknowledgements}

This work was supported by the NRF funded by the MSIP of the Korean government (Grant No. 2006-0093853) and IBS-R009-D1. Computations were supported by the Korea Institute of Science and Technology Information.

\section{References}

1 L. Schlapbach and A. Züttel, Nature, 2001, 414, 353-358.

2 B. Sakintuna, F. Lamari-Darkrim and M. Hirscher, Int. J. Hydrogen Energy, 2007, 32, 1121-1140.

3 L. J. Murray, M. Dincă and J. R. Long, Chem. Soc. Rev., 2009, 38, 1294-1314.

4 M. P. Suh, H. J. Park, T. K. Prasad and D.-W. Lim, Chem. Rev., 2011, 112, 782-835.

5 M. Dinca, A. Dailly, Y. Liu, C. M. Brown, D. A. Neumann and J. R. Long, J. Am. Chem. Soc., 2006, 128, 16876-16883.

6 E. Durgun, S. Ciraci, W. Zhou and T. Yildirim, Phys. Rev. Lett., 2006, 97, 226102.

7 G. J. Kubas, J. Organomet. Chem., 2001, 635, 37-68.

8 W.-Q. Deng, X. Xu and W. A. Goddard, Phys. Rev. Lett., 2004, 92, 166103.

9 G. K. Dimitrakakis, E. Tylianakis and G. E. Froudakis, Nano Lett., 2008, 8, 3166-3170.

10 E. Tylianakis, G. M. Psofogiannakis and G. E. Froudakis, J. Phys. Chem. Lett., 2010, 1, 2459-2464. 
11 J. W. Burress, S. Gadipelli, J. Ford, J. M. Simmons, W. Zhou and T. Yildirim, Angew. Chem., Int. Ed., 2010, 49, 8902-8904.

12 S. S. Han and S. S. Jang, Chem. Commun., 2009, 5427-5429.

13 R. B. Getman, J. H. Miller, K. Wang and R. Q. Snurr, J. Phys. Chem. C, 2011, 115, 2066-2075.

14 E. Klontzas, A. Mavrandonakis, E. Tylianakis and G. E. Froudakis, Nano Lett., 2008, 8, 1572-1576.

15 C. Kittel and H. Kroemer, Thermal Physics, W. H. Freeman, New York, 2nd edn, 1980.

16 R. S. Aga, C. L. Fu, M. Krčmar and J. R. Morris, Phys. Rev. B: Condens. Matter Mater. Phys., 2007, 76, 165404.

17 S. Patchkovskii, S. T. John, S. N. Yurchenko, L. Zhechkov, T. Heine and G. Seifert, Proc. Natl. Acad. Sci. U. S. A., 2005, 102, 10439-10444.

18 H.-K. Jeong, Y. P. Lee, R. J. Lahaye, M.-H. Park, K. H. An, I. J. Kim, C.-W. Yang, C. Y. Park, R. S. Ruoff and Y. H. Lee, J. Am. Chem. Soc., 2008, 130, 1362-1366.

19 T. H. Kim, J. Bae, T. H. Lee, J. Hwang, J. H. Jung, D. K. Kim, J. S. Lee, D. O. Kim, Y. H. Lee and J. Ihm, Nano Energy, 2016, 27, 402-411.

20 P. Hohenberg and W. Kohn, Phys. Rev., 1964, 136, B864.

21 W. Kohn and L. J. Sham, Phys. Rev., 1965, 140, A1133.

22 J. P. Perdew, K. Burke and M. Ernzerhof, Phys. Rev. Lett., 1996, 77, 3865.

23 S. Grimme, J. Comput. Chem., 2006, 27, 1787-1799.

24 G. Kresse and J. Furthmüller, Phys. Rev. B: Condens. Matter Mater. Phys., 1996, 54, 11169.
25 I. F. Silvera and V. V. Goldman, J. Chem. Phys., 1978, 69, 4209-4213.

26 K. A. Mkhoyan, A. W. Contryman, J. Silcox, D. A. Stewart, G. Eda, C. Mattevi, S. Miller and M. Chhowalla, Nano Lett., 2009, 9, 1058-1063.

27 R. Lahaye, H. Jeong, C. Park and Y. Lee, Phys. Rev. B: Condens. Matter Mater. Phys., 2009, 79, 125435.

28 D. W. Boukhvalov and M. I. Katsnelson, J. Am. Chem. Soc., 2008, 130, 10697-10701.

29 M. C. Nguyen, M.-H. Cha, K. Choi, Y. Lee and J. Ihm, Phys. Rev. B: Condens. Matter Mater. Phys., 2009, 79, 233408.

30 G. Henkelman, A. Arnaldsson and H. Jónsson, Comput. Mater. Sci., 2006, 36, 354-360.

31 D. Frenkel and B. Smit, Understanding Molecular Simulation: From Algorithms to Applications, Academic Press, San Diego, Calif, 2nd edn, 2001.

32 C. Robledo, M. Rojas, O. Camara and E. Leiva, Int. J. Hydrogen Energy, 2014, 39, 4396-4403.

33 A. Sigal, M. Villarreal, M. Rojas and E. Leiva, Int. J. Hydrogen Energy, 2014, 39, 5899-5905.

34 C. P. Herrero and R. Ramírez, Phys. Rev. B: Condens. Matter Mater. Phys., 2010, 82, 174117.

35 A. Sigal, M. Rojas and E. Leiva, Phys. Rev. Lett., 2011, 107, 158701.

36 J. Hwang, C. Park, K. Choi, M.-H. Cha, R. Ahuja, D. W. Kim, D. O. Kim, K. Sagong, U. G. Joung and H. Jeong, Nano, 2012, 7, 1250044 . 